\title{
7. Über die innere Reibung verdünnter Gase und uber den Zusammenhang \\ der Gleitung und des Temperatursprunges an der Grenze zwischen Metall und Gas ${ }^{1}$ ); \\ von A. Timiriazeff.
}

\section{§ 1. Finleitung.}

Die von Kundt und Warburg ${ }^{2}$ ) entdeckte Abnahme des Wärmetransports bei kleinen Drucken kann, wie die Untersuchungen von $\mathrm{Smoluchowski}$ ) gezeigt haben, durch die Annahme erklärt werden, daB an der Grenze zwischen der GefäBwand und Gas eine Temperaturdifferenz sich bildet. Die experimentell bestimmte GröBe des "Temperatursprunges" ist im Einklang mit der Theorie von Smoluchowskis) umgekehrt proportional dem Gasdrucke, wie es aus den Messungen von Smoluchowski ${ }^{5}$ ), Gehrcke ${ }^{6}$ ) und Lasareff ) hervorgeht.

Andererseits haben die Untersuchungen von $\mathrm{Kundt}$ und Warburg ${ }^{8}$ ) über die innere Reibung der Gase gezeigt, daB die bei kleinen Gasdrucken auftretende Abnahme des BewegungsgröBentransports durch die Annahme eiuer Gleitung längs der Oberfläche fester Körper erklärt werden kann. Die Größe der Gleitung ist auch dem Drucke umgekehrt proportional.

1) Ein kurzer Bericht über diese Untersuchung wurde auf dem II. Mendelejeffschen KongreB in St. Petersburg am 25. Dezember 1911 vorgetragen [vgl. Tageblatt des Kongresses Nr. 8. p. 68 (russisch)].

2) A. Kundt u. E. Warburg, Pogg. Ann. 156. p. 177. 1875.

3) M. v. Smoluchowski, Wied. Ann. 64. p. 101. 1898.

4) M. v. Smoluchowski, Sitzungsber. d. Akad. d. Wiss. zu Wien 107. Abt. II a. p. 304. 1898; 108. Abt. II a. p. 5. 1899.

5) M. v. Smoluchowski, l. e.

6) E. Gehrcke, Ann. d. Phys. 2. p. 102. 1900.

7) P. Lasareff, Ann. d. Phys. 37. p. 233. 1912.

8) A. Kundt u. E. Warburg, Pogg. Ann. 155. p. 337, 525. 1875. 
Die Aufgabe der vorliegenden Untersuchung war, die Reibungserscheinungen bis zu großen Verdünnungen $(0,001 \mathrm{~mm})$ zu verfolgen und den Zusammenhang der Wärmeleitungs- und Reibungsphänomene in verdünntem Gase zu ermitteln - einen Zusammenhang, der eine Ergänzung der wohlbekannten Beziehung zwischen der inneren Reibung und Wärmeleitung bei mittleren Druckverhältnissen bildet.

\section{§ 2. Methode.}

Es war die Methode der stationären Ablenkung gewählt; konzentrisch und im Innern eines rotierenden Zylinders $A$ (Fig. 1) war ein Zylinderkörper $B$ mittels eines dünnen Metalldrahtes (Phosphorbronze 0,1 mm) aufgehängt.

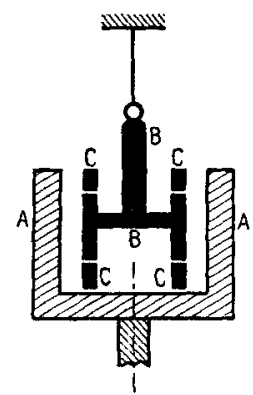

Fig. 1. Die Ablenkung wurde durch Spiegelablesung nach der üblichen Art beobachtet. Zwei Schutzringe $C C$ ermöglichten, von der Randkorrektion abzusehen. Die passend gewählte elektromagnetische Dämpfung gestattete eine rasche Einstellung der stationären Ablenkung. Die Umdrehungsgeschwindigkeit wurde mittels Tourenzähler und Stoppuhr bestimmt.

Ähnliche Methoden sind vielfach bei den Reibungsuntersuchungen angewandt worden (vgl. z. B. La Couette ${ }^{1}$ ), Gyözö Zemplen ${ }^{2}$ ), Drew ${ }^{3}$ ) und Gilchrist $\left.{ }^{4}\right)$ ) und baben den Vorzug vor den von $\mathrm{Kund} t$ und Warburg ${ }^{5}$ ), Crookes ${ }^{6}$ ) und Hogg ${ }^{7}$ ) angewandten Methode der Dämpfungsdekrementbestimmung, daB man keiner Randkorrektion und keiner Korrektion wegen der Dämpfung des Aufhängedrahtes bedarf, und daB die Beobachtungen viel weniger Zeit beanspruchen.

1) La Couette, Journ. de Phys. 9. 2. Ser. p. 414. 1890.

2) Gyözö Zemplén, Ann. d. Phys. 38. p. 71. 1912.

3) E. R. Drew, Phys. Rev. 12. p. 114. 1901.

4) L. Gilchrist, Physik. Zeitschr. 14. p. 160. 1913.

5) A. Kundt u. E. Warburg, 1. e.

6) Sir William Crookes, Phil. Trans. of the Roy. Soc. London 172. p. 387. 1882 .

7) J. L. Hogg, Contributions from the Jefferson Physical Laboratory 7. 1909. 
Der ganze Apparat war unter eine Glasglocke gestellt und der Transport der BewegungsgröBe durch die Gasschicht zwischen den Zylindern $A$ und $B$ (Fig. 1) von Atmosphärendruck bis zu $0,001 \mathrm{~mm}$ untersucht.

Um den EinfluB der Gasschichtdicke zu ermitteln, war eine Reihe von Zylindern $A$ von verschiedenem inneren Durchmesser hergestellt; diese Zylinder konnte man leicht auswechseln.

\section{§ 3. Beschreibung der Apparate.}

Der Reibungsapparat war auf einer Stahlscheibe $A A$ (Fig. 2) (von $200 \mathrm{~mm}$ Durchmesser) montiert. In der Scbeibe war eine ringförmige Rinne ausgedreht, an welche die Glasglocke $B B$ angeschliffen war (der Schliff war mit Ramsayfett gedichtet und von auBen durch Quecksilber verschlossen). Die $920 \mathrm{~mm}$ lange Stahlachse $a a$ geht im Innern eines in der Mitte der Grundscheibe eingeschraubten Stahlrohres $C$ durch und trägt ein mit Quecksilber gefülltes Gefäß $G$. Die ganze Vorrichtung bildet einen BarometerverschluB und erlaubt zugleich, die Achse von auBen zu drehen. Am unteren Ende ruht die Achse auf einer Stahlkugel in der aus der Fig. 2 ersichtlichen Weise.

Am oberen Ende der Achse ist eine Scheibe $M$ angebracht, welche die auswechselbaren Zylinder $P P$ trägt. Das obere Lager der Achse (Kugellager), das zugleich den unteren Schutzring bildet, ist durch vier Schrauben an die vier durch die Öffnungen in $R$ hervorragenden Teile des oberen Gestelles des Apparates $Q$ befestigt.

Der aufgehängte Zylinderkörper $R$ hat, auBer den genannten vier Öffnungen, durch welche die vier herrorragenden Teile $Q$ frei durchgehen und die den für die Ablenkungen nötigen Spielraum zulassen, noch vier kleine Öffnungen $b$, welche mit entsprechenden Löchern, die in das Gestell $Q$ gebohrt sind (in der Fig. 2 nicht gezeigt), direktes Beschauen der Teile $R$ und $a$ des aufgehängten Körpers bzw. der rotierenden Achse zulassen und eine genaue Justierung des Apparates ermöglichen; diese Justierung kann ausgefuhrt werden mittels dreier Stellschrauben, die an dem den Torsionskopf tragenden Teil $E$ angebracht sind. 
Das den Torsionskopf tragende Messingrohr $K$ hat am unteren Ende vier Ausschnitte, die das Anbringen einer silbernen Scheibe $s s$ am oberen Teile des aufgehängten Körpers $R$

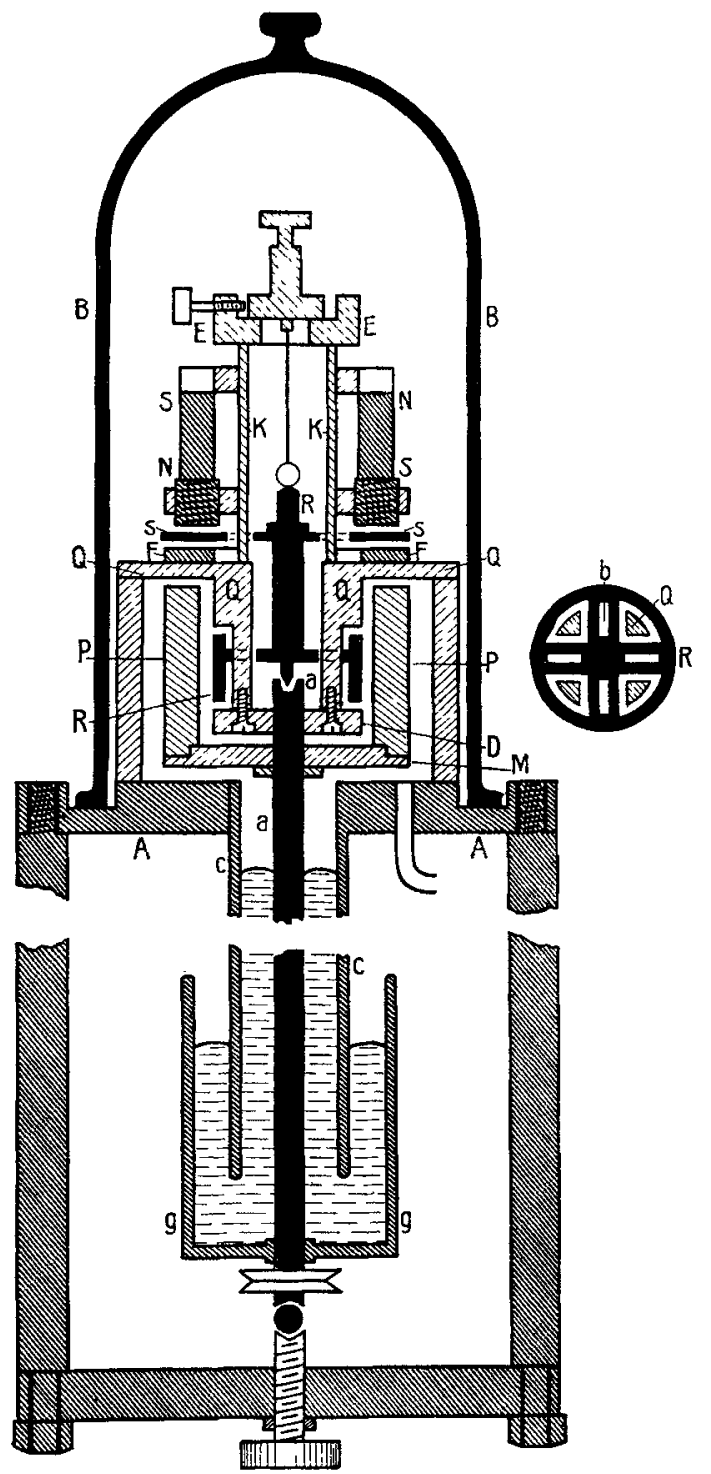

Fig. 2. 
ermöglichen. Die Scheibe $s s$ hat vier Ausschnitte, die einen für die Ablenkung nötigen Spielraum zulassen. Diese Scheibe dient als Dämpfungsvorrichtung; vier Stahlmagnete $N S$ mit verstellbaren Polen sind am Messingrohr $K$ befestigt; der magnetische KraftfluB wird durch einen an $Q$ liegenden sternförmigen Eisenkörper $F$ ergänzt. Die silberne Scheibe war aus reinem Silber hergestellt und nachher nach Kohlrausch ${ }^{1}$ ) mit verdünnter kochender Schwefelsäure gewaschen und dann sorgfältig mit kochendem Wasser gespült, um vom ,magnetischen Staub" frei zu werden. Die so verfertigte Dämpfungsscheibe war folgendermaßen auf magnetische Eigenschaften geprufut: es war eine Beobachtungsreihe mit Dämpfung ausgeführt und mit einer entsprechenden Reibe verglichen, die ohne Dämpfung ausgeführt war. Es stellte sich heraus, daB die Dämpfung keinen merklichen Einflub auf die Ausschläge hat. Wäscht man nicht mit Schwefelsäure, so bekommt man eine erhebliche Unsymmetrie der Ablenkung und die Ausschläge sind von 4 bis 2 Proz. kleiner, was einer Magnetisierung der oberflächlichen Verunreinigungen zugeschrieben werden muB.

Der ganze Apparat war, außer den genannten eisernen und stählernen Teilen, aus Messing hergestellt und vernickelt. Der Druck wurde mittels Mc Leod schen Manometers gemessen. Trockene Gase, Luft, Kohlensäure und Wasserstoff (die beiden letzten aus käuflichen Stahlzylindern entnommen) wurden in den Apparat eingelassen und die Versuche bis $10 \mathrm{~mm}$ ausgeführt; nachdem wurden die beiden Quecksilberverschlüsse $V_{1}$ und $V_{2}$ (Fig. 3) geschlossen. Die im GefäBe $R$ enthaltene Gasmenge dient für weitere Versuche bei größeren Verdünnungen; der Apparat wurde mit einer Gaedepumpe evakuiert und dann mittels zweier Verschlüsse $F_{1}$ und $V_{2}$ kleine Gasportionen eingeführt. Diese Vorrichtung ${ }^{2}$ ) ermöglicht eine rasche Einstellung beliebiger Drucke.

Die Achse war durch einen Drehstrommotor (1400 Touren $1 / 4$ P.S.) über ein Zahnradrorgelege $(1: 2 \overline{5})$ angetrieben. An der Achse des Apparates war mittels Zahnrädern ein Touren-

1) F. Kohlrausch, Lehrbuch der praktisehen Physik, 9. Auf. 1901. p. 319.

2) Vgl. P. Lasareff, l. c. p. 238. 
zähler angebracht, der nach einer bestimmten Tourenzahl ein Läutewerk in Gang setzte, was eine bequeme Geschwindigkeitsmessung mit Hilfe einer Stoppuhr gestattet.

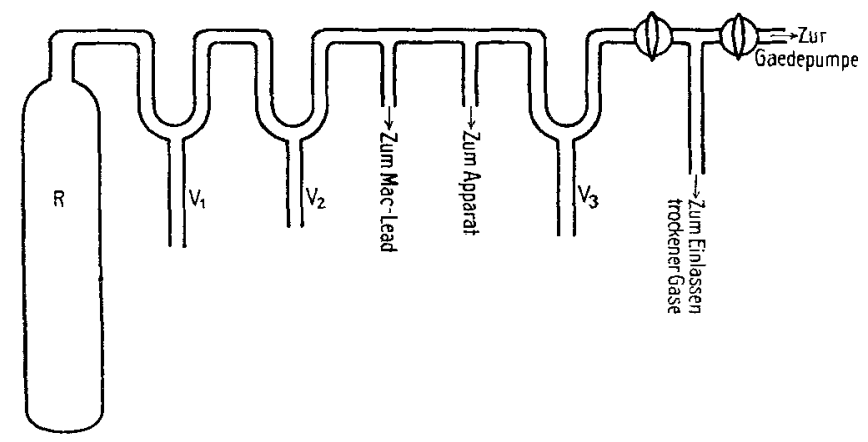

Fig. 3.

\section{§4. Zur Theorie.}

Die Theorie der Reibungserscheinung in stark verdünnten Gasen war für den Fall, daB die mittlere freie Weglänge nicbt mehr verschwindend klein im Vergleich zum Abstand der festen Körper ist, die durch eine Gasschicht die BewegungsgröBe übertragen, zuerst von Kundt und Warburg ${ }^{1}$ ) entwickelt. Die genannten Forscher haben gezeigt, daS an der Oberfläche der festen Körper eine Gleitung stattfindet, und daB diese Gleitung umgekehrt proportional der Gasdichte ist, deswegen ist die Gleitung nur bei großen Verdünnungen zu beobachten. Bei dieser gastheoretischen Untersuchung waren die genannten Forscher von den üblichen Vorstellungen der elastischen Kugeln ausgegangen und haben die Theorie der Erscheinung an der Grenze zwischen Gas und festem Körper nach einem sinnreichen Näherungsverfahren entwickelt.

M. v. Smoluchowski hat in seiner ersten ${ }^{2}$ ) theoretischen Abhandlung über den Temperatursprung denselben Weg eingeschlagen, später ${ }^{3}$ ) aber hat er gezeigt, daB man zum gleichen

1) A. Kundt u. E. Warburg, Pogg. Ann. 155. p. 340. 1875.

2) M. v. Smoluchowski, Sitzungsber. d. Akad. d. Wiss, zu Wien 107. Abt. II a, p. 304. 1898.

3) M. r. Smoluchowski, l. c. 108. Abt. IIa. p. 5. 1899. 
Resultat gelangen kann, wenn man ron der strengeren Maxwellschen Auffassung der Gastheorie ausgeht, wobei das mühsame Näherungsverfahren wegfällt.

Wir können ohne weiteres diese strengere Maxwell sche Theorie auch auf Reibungsvorgänge anwenden. Boltz$\left.\operatorname{mann}^{1}\right)$ hat gezeigt, daB in der Gasschicht zwischen zwei parallelen Ebenen, von denen die eine $A$ (Fig. 4) ruht und die andere in ihrer Ebene sich geradlinig und gleichförmig bewegt, die Ge. schwindigkeitsverteilung durch die folgende Funktion ausgedrückt werden kann

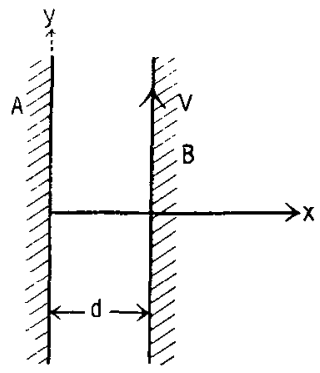

Fig. 4.

$$
\left\{\begin{array}{l}
F(\xi, \eta, \zeta, x) \\
=A\left\{1+2 h m \frac{\partial v}{\partial x} x \eta-2 h m \frac{\partial v}{\partial x} \frac{\mu}{p} \eta \xi\right\} e^{-h m\left(\xi^{2}+\eta^{2}+\xi^{2}\right)}
\end{array}\right.
$$

wo

$\xi, \eta, \zeta$ die Geschwindigkeitskomponenten der Moleküle sind,

$v$ die Geschwindigkeit der Gasmasse parallel zur $y$-Achse,

$x$ die $x$-Koordinate der Gasschicht, von der ruhenden Platte $A$ aus gerechnet,

$p$ der Gasdruck,

$\mu$ die Reibungskonstante,

$m$ die Masse des Moleküls,

$A$ und $h$ Konstanten, wobei $h$ durch die wohlbekannte Relation $\overline{c^{2}}=\frac{3}{2 h m}$ mit dem mittleren Quadrat der Molekulargeschwindigkeit $\overline{e^{2}}$ verknüpft ist.

Diese Formel gilt nưr im Falle, daB man die höheren Potenzen der Größen

$$
2 h m \frac{\partial v}{\partial x} \quad \text { und } \quad 2 h m \frac{\partial v}{\partial x} \frac{\mu}{p}
$$

vernachlässigen kann. Eine Bedingung, die in unseren Versuchen mit Geschwindigkeiten, die klein sind im Vergleich zum mittleren Quadrat der Molekulargeschwindigkeit, erfüllt ist (maximale Umfangsgeschwindigkeit $16 \mathrm{~cm} / \mathrm{sec}$, Maximalwert $\operatorname{der}$ GröBe $\left.\partial v / \partial x=270 \sec ^{-1}\right)$. Wenn wir weiter annehmen,

1) L. Boltzmann, Weitere Studien über das Wărmegleichgewicht unter Gasmolekz̈len. Sitzungsber. d. Akad. d. Wiss. zu Wien 66. p. 275 bis 370. 1872; Wissensch. Abhandl. I B. p. 362. Leipzig 1909.

Annalen der Physik. IV. Folge. 40. 
daB an den Ebenen $A$ und $B$ die von Smoluchowski benutzten Maxwellschen Grenzbedingungen statthaben, so bekommen wir die Grundgleichungen unseres Problems. Die erste Grenzbedingung ${ }^{1}$ ):

$$
\varrho_{2} \bar{\xi}_{2}=-(1-f) \varrho_{1} \bar{\xi}_{1}+f \varrho_{2}^{\prime} \bar{\xi}_{2}^{\prime},
$$

wo $f$ der Anteil ,absorbierter" Moleküle, die später von der Platte wieder ,emittiert" werden, ist; $(1-f)$ bezieht sich auf Moleküle, die nach den Gesetzen des elastischen StoBes reflektiert werden; $\varrho$ ist die Dichte und $\xi$ die mittlere Geschwindig. keit in der Richtung der $x$-Achse. Die Indizes 2 beziehen sich auf Moleküle, die von der Wand $A$ in positiver $x$-Richtung fliegen, ebenso wie die Indizes 1 auf Moleküle, die im entgegengesetzten Sinne sich bewegen.

Die Gleichung sagt aus, daB die von der Wand fliegenden Moleküle aus ,reflektierten" und „emittierten" Molekülen bestehen; für die "emittierten" nehmen wir an, daB sie die Maxwellsche Geschwindigkeitsverteilung besitzen.

Ganz analog kann man die zweite Grenzbedingung für die BewegungsgröBe schreiben $\left.{ }^{2}\right)$ :

$$
\varrho_{2} \overline{\xi_{2} \eta_{2}}=-(1-f) \varrho_{1} \xi_{1} \eta_{1}+f \varrho_{2}{ }^{\prime} \xi_{2}^{\prime} u,
$$

wobei $u$ die relative Geschwindigkeit der Platte und der angrenzenden Gasschicht ist.

Führen wir die in (2) und (3) angedeuteten Rechnungen mit Hilfe von (1) aus und bemerken dabei, daB die Integration nach $\xi$ für die Größen mit Index 2 von 0 bis $\infty$ und für Größen mit (1) von $-\infty$ bis 0 ausgeführt werden soll, so bekommen wir, nach Division der Gleichung (3) durch (2), die Gleichung:

$$
u=-\frac{2-f}{f} \frac{1}{2} \sqrt{\frac{\pi}{h m}} \frac{\mu}{p} \frac{\partial v}{\partial x} .
$$

Oder durch Ersatz von $h m$ durch die absolute Temperatur $T$, das Molekulargewicht $M$ und die Gaskonstante $R_{0}$ :

$$
u=-\frac{2-f}{f} \frac{\mu}{p} \sqrt{\frac{\pi R_{0} T}{2 M}} \frac{\partial v}{\partial x} .
$$

1) J. C. Maxwell, Scientific Papers 2. p. 707, Gleichung (61).

2) J. C. Maxwell, l. c. p. 707, Gleichung (62). 
Wie man sieht, ist $\left(4^{\prime}\right)$ ganz analog der Grenzbedingung einer Flüssigkeit mit Reibung unter Berücksichtigung einer endlichen Gleitung:

$$
k u^{\prime}=\mu \frac{\partial v}{\partial x},
$$

wo $u^{\prime}=-u$ ist.

Nur sagt die hydrodynamische Theorie über die Konstanten $k$ und $\mu$ nichts weiter aus; während die nach der Maxwellschen Theorie berechnete Beziehung ${ }^{1}$ ), in Übereinstimmung mit $\mathrm{Kundt}$ und Warburg ${ }^{2}$ ), zeigt, daB die GröBe $\mu / k$ umgekehrt proportional dem Drucke $p$ ist.

Nach der hydrodynamischen Theorie ist die pro Quadratzentimeter in der Zeiteinheit übertragene Bewegungsgröße gleich $\left.^{3}\right)$ :

$$
G=\frac{\mu V}{d+2 \frac{\mu}{k}},
$$

wo $V$ die Geschwindigkeit der Platte $B$ (Fig. 4) (die Geschwindigkeit der Platte $A$ ist gleich Null angenommen) und $d$ der Plattenabstand ist. Führen wir statt $\mu / k$ den Ausdruck

$$
\frac{\mu}{k}=\frac{2-f}{f} \frac{\mu}{p} \sqrt{\frac{\pi R_{0} T}{2 M}}
$$

aus (4') in (6) ein und setzen zur Abkürzung

so bekommen wir

$$
\frac{2-f}{f} \mu \sqrt{\frac{\pi R_{0} T}{2 M}}=a_{0},
$$

$$
G=\frac{\mu V}{d+2 \frac{a_{0}}{p}} .
$$

Wegen der Kleinheit der Größe $a_{0}$ bei groBen Werten von $p$ ist das Glied $2 a_{0} / p$ im Vergleich zu $d$ verschwindend klein. Die Größe $G$ ist in diesem Stadium unabhängigf rom Drucke $p$, in Übereinstimmung mit dem Maxwellschen Gesetze.

1) J. C. Maxwell, l. c. p. 708, Gleichung (69).

2) A. Kundt u. E. Warburg, l. c.

3), M. Brillouin, Leçons sur la Viscosité p. 50. Paris, GauthierVillars 1907. 
Bei kleinen Werten von $p$ überwiegt das zweite Glied im Nenner $\left(6^{\prime}\right)$ und wir bekommen

$$
G=\frac{\mu V}{2 a_{0}} p
$$

Die Größe $G$ ist dem Drucke proportional.

Wegen der GröBe des Druckintervalles (760 mm - 0,001 mm) ist es zweckmäBiger, den Logarithmus des Druckes $x\left(p=e^{x}\right)$ in die Formel einzuführen

$$
G=\frac{\mu V e^{x}}{d \cdot e^{x}+2 a_{0}} .
$$

Aus dieser Gleichung folgt, daB die Kurve $G=f(x)$ einen Inflexionspunkt für

hat, was für $G$ den Wert

$$
e^{x}=\frac{2 a_{0}}{d}
$$

$$
G=\frac{\mu V}{2 d}
$$

gibt, i. e. gleich der Hälfte der Größe $G$ für Normaldruck.

Führen wir in die Gleichung (7) den der mittleren Weglänge in der Maxwell schen Theorie entsprechenden Wert ${ }^{1}$ $\lambda=$ Relaxationszeit $\times$ mittlere Geschwindigkeit ein, so erhalten wir

$$
\frac{a_{0}}{p}=\frac{2-f}{f} \frac{2}{\pi} \lambda
$$

Die Konstante $a_{0}$ ist also mit der Temperatursprungkonstante ron Smoluchowskiª):

$$
\gamma=\frac{15}{4 \pi} \frac{2-f}{f} \lambda
$$

durch folgende Bedingung verknüpft:

$$
a_{0}=\frac{8}{15} \gamma p \text {. }
$$

Wir können somit die Konstante $a_{0}$ aus den Messungen des Temperatursprunges berechnen und die so berechnete Kurve $G=f(p)$ mit dem Experiment direkt vergleichen.

Es muB noch hervorgehoben werden, daB Formel $\left(6^{\prime \prime}\right)$ für Weglängen, die groB im Vergleich mit dem Plattenabstand

1) J. C. Maxwell, l. c. p. 681.

2) M. v. Smoluchowski, Sitzungsber. d. Akad. d. Wiss. zu Wien 108. Abt. II a. 1909. Gleichung (11). 
sind, auf direkte Weise abgeleitet werden kann unter Vernachlässigung der gegenseitigen molekularen Zusammenstöß日 in der Gasschicht. Die Rechnung, die vollkommen analog ist mit der von Smoluchowski für die Wärmeleitung durchgeführten ${ }^{1}$ ), ergibt:

$$
G=\frac{f}{2-f} v \sqrt{\frac{M}{2 \pi R_{0} T}} \cdot p,
$$

was mit dem Ausdruck (6") unter Berücksichtigung von (7) übereinstimmt.

Wir müssen noch die Korrektion berücksichtigen, welche von der endlichen Krümmung der von uns angewandten Zylinderkörper herrührt; diese Korrektion fällt im gröBten Teile klein aus wegen der Kleinheit von $R_{8}-R_{1}$ im Vergleich zu $R_{1}$ und $R_{2}$.

Die hydrodynamische Theorie fuhrt, wenn man die innere Reibung der Flüssigkeiten berücksichtigt, zu folgenden Resultaten, die für den Fall konzentrischer Zylinder gelten (diese Resultate gelten auch für Gase); die Winkelgeschwindigkeit der rotierenden Flüssigkeit $\omega$ ist durch den Ausdruck:

$$
\omega=\frac{A}{r^{2}}+B
$$

gegeben, wo $A$ und $B$ Konstanten sind. Nimmt man die Winkelgeschwindigkeit der Gasschicht an der Grenze des inneren bzw. äuBeren Zylinders mit den Radien $R_{1}, R_{2}$ gleich $\omega_{1}$ bzw. $\omega_{2}$ an, so bekommen wir:

$$
A=\frac{R_{1}^{2} R_{2}^{2}}{R_{2}^{2}-} \frac{R_{1}^{2}}{{ }^{2}}\left(\omega_{2}-\omega_{1}\right) \text {. }
$$

Die Geschwindigkeit der Scherung ist gleich $r(d \omega) / d r$; deshalb ist das gesuchte Moment der tangentialen Kräfte, die auf die innere zylindrische Oberfläche vom Radius $R_{1}$ wirken, pro Längeneinheit gleich

$$
\left.G=4 \pi \mu \frac{R_{1}{ }^{2} R_{2}{ }^{2}}{R_{2}{ }^{2}-R_{1}{ }^{2}}\left(\omega_{2}-\omega_{1}\right) \cdot{ }^{3}\right)
$$

1) M. v. Smoluchowski, Phil. Mag. 21. p. 11. 1911.

2) H. Lamb, Lehrbuch der Hydrodynamik p. 676, Gleichung (2).

3) H. Lamb, l. c. p. 676, Gleichung (6). 
Findet an der Oberfläche eine Gleitung statt, und nehmen wir an, daB die ,äuBere" Reibung der relativen Geschwindigkeit $u^{\prime}$ proportional ist, so finden wir:

$$
\left.T=k u^{\prime},{ }^{1}\right)
$$

wo $T$ die Tangentialkraft (in unserem Falle $T=\mu r(d \omega) / d r$ ), $k$ die "Konstante" der äuBeren Reibung und $u^{\prime}$ die relative Geschwindigkeit der Flüssigkeit und der festen Wand ist.

Nennen wir die Winkelgeschwindigkeiten der festen Zylinder $\omega_{1}^{\prime} \mathrm{bzw} . \omega_{2}^{\prime}$, so bekommen wir als Grenzbedingungen

(16) $\mu\left(\frac{d \omega}{d r}\right)_{R_{1}}=k\left(\omega_{1}-\omega_{1}{ }^{\prime}\right)$ und $\mu\left(\frac{d \omega}{d r}\right)_{R_{2}}=k\left(\omega_{2}{ }^{\prime}-\omega_{2}\right)$.

Führen wir in (16) die aus (12) und (13) folgenden Werte von $d \omega / d r$ ein und addieren die beiden Gleichungen (16), so erhalten wir:

$$
\omega_{2}-\omega_{1}=\frac{\omega_{2}^{\prime}-\omega_{1}^{\prime}}{1+2 \frac{\mu}{k} \frac{1}{R_{1} R_{2}} \frac{R_{1}^{\mathrm{s}}+R_{2}^{\mathrm{s}}}{R_{2}{ }^{2}-R_{1}{ }^{2}}} .
$$

Führen wir weiter diese GröBe in (14) ein und bemerken, $\mathrm{daB} \quad \omega_{1}{ }^{\prime}=0$ ist, so finden wir die folgende Lösung unseres Problems:

$$
\text { (18) } \left.G=2 \pi R_{1}{ }^{2} R_{2} \omega_{2}^{\prime} \mu \frac{2 R_{2}}{R_{1}+R_{2}} \frac{1}{\left(R_{2}-R_{1}\right)+2 \frac{\mu}{k} \frac{R_{1}{ }^{3}+R_{2}{ }^{3}}{R_{1} R_{2}\left(R_{1}+R_{2}\right)}} .^{2}\right)
$$

Vergleicht man dieses Resultat mit der Gleichung (6), die für eine unendliche Ebene abgeleitet war, so sieht man unmittelbar, daB hier zwei Faktoren:

$$
\left.\frac{2 R_{2}}{R_{2}+R_{1}} \text { und } \frac{R_{1}{ }^{3}+R_{2}{ }^{3}}{R_{1} R_{2}\left(R_{1}+R_{2}\right)} \text { (als Faktor bei } 2 \frac{\mu}{k}=2 \frac{a_{0}}{p}\right)
$$

auftreten.

Es muB besonders betont werden, daB auBer den in der hydrodynamischen Theorie gemachten Annahmen die Formel (18) auf der Annahme beruht, daB $\mu / k=a_{0} / p$ auch hier gilt, d. h. daß die äuBere Reibung unabhängig von der Krümmung der Flächen ist.

1) M. Brillouin; l. c. p. 44.

2) (18) ist auf die Längeneinheit bezogen; die Höhe des Zylinders war $1,995 \mathrm{~cm}$. 
$\mathrm{DaB}$ (18) nicht die strenge Lösung darstellt, sieht man schon daraus, daB für große Verdünnungen, wenn man $R_{2}-R_{1}$ vernachlässigen kann, die GröBe $G$ einen Faktor

$$
\frac{2 R_{1} R_{2}^{2}}{R_{1}^{3}+\bar{R}_{2}^{3}}
$$

hat; wenn man aber für den Fall rotierender Zylinder einen der Formel (11) entsprechenden Ausdruck für große mittlere Weglängen ableitet, so findet man den oben genannten Faktor $=1$.

Diese Abweichung kann man wegen ihrer Kleinheit aus unseren Versuchen nicht finden, weshalb hier auch die entsprechenden Rechnungen nicht ausgeführt worden sind.

\section{\$ 5. Die Beobachtungen.}

Die erste Messungsreihe war mit Luft ausgeführt, und zwar mit vier verschiedenen äußeren Zylindern, deren Durchmesser so gewählt waren, daß die GröBen $R_{2}-R_{1}$ ungefähr eine geometrische Reihe bildeten:

$$
\begin{aligned}
& \text { Zylinder Nr. } 1 \quad R_{2}-R_{1}=0,406 \mathrm{~cm}
\end{aligned}
$$

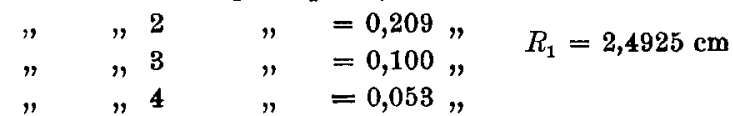

Es stellte sich heraus, daB bei kleinen Umdrehungsgeschwindigkeiten, die benutzt wurden $(0,3$ bis 0,86 Umdrehungen pro sec), die Ablenkungen innerhalb der Beobachtungsfehler der Umdrehungszahl proportional waren, was aus der folgenden Tabelle unmittelbar zu ersehen ist.

Tabelle 1.

Zylinder Nr. 3.

\begin{tabular}{c|c|c}
\hline \hline$\frac{\mathrm{Umdr} .}{\mathrm{sec}}$ & $\alpha$ Ausschlag & $\frac{\alpha}{u}$ \\
\hline \hline 0,854 & 121,4 & 142,8 \\
0,614 & 87,5 & 142,5 \\
0,325 & 46,4 & 142,6
\end{tabular}

Der Absolutwert der Reibungskonstanten war durch Bestimmung der Direktionskraft des Drahtes an Körpern von 
bekanntem Trägheitsmoment in der üblichen Weise ermittelt. Dabei war darauf geachtet, daB die Trägheitskörper von demselben Gewicht wie der aufgehängte Zylinder waren (ca. $130 \mathrm{~g}$ ), um den von den elastischen Eigenschaften des Aufhängedrahtes abhängigen Wert der Direktionskraft unter gleichen Belastungsbedingungen wie bei den ausgeführten Versuchen zu ermitteln. Diese Messungen ergaben:

Direktionskraft: $D=37,75 \mathrm{~g} \mathrm{~cm}^{2} \mathrm{sec}^{-2}$,

Reibungskonstante für Luft: $\mu=0,000198 \mathrm{~g} \mathrm{~cm}^{-1} \mathrm{sec}^{-1}$.

Für Kohlensäure und Wasserstoff ergaben sich folgende Werte ( $\mu$ Luft $=1$ angenommen)

$$
\begin{aligned}
& \frac{\mu_{\mathrm{CO}_{2}}}{\mu_{\mathrm{Luft}}}=0,855, \\
& \frac{\mu_{\mathrm{H}_{2}}}{\mu_{\mathrm{Luft}}}=0,530 .
\end{aligned}
$$

In befriedigender Übereinstimmung mit den früheren zahlreichen Beobachtungen, die nach rerschiedenen Methoden und durch verschiedene Autoren ausgeführt waren.

Wir gehen jetzt zur Beschreibung der Hauptversuche über. Jede Versuchsreihe war in folgender Weise ausgeführt: es war ein gegebener Druck hergestellt, die Nullage notiert, der Motor in Gang gesetzt und der stationäre Ausschlag abgelesen; wegen der Dämpfung (vgl. oben § 3) stellt sich der Ausschlag innerhalb $50 \mathrm{sec}$ ein und bleibt vollkommen konstant, abgesehen von seltenen Schwankungen innerhalb 0,1 Skalenteile. Die Umdrehungsgeschwindigkeit wurde auf die oben angeführte Art bestimmt.

Die folgende Tabelle zeigt ein Beispiel der Beobachtungsprotokolle:

$$
\text { Tabelle } 2 .
$$

Versuchsreihe Nr. 36. Zylinder Nr. $2 . \quad p=750 \mathrm{~mm}$.

Skalenabstand $1085 \mathrm{~mm}$.

\begin{tabular}{c|c|c|c|c|c|c|c|c|c|c|c}
\hline \hline 0 & $\alpha_{1}$ & 0 & $\alpha_{2}$ & 0 & $\alpha_{1}$ & 0 & $\alpha_{9}$ & 0 & $\alpha_{2}$ & 0 & $\alpha_{3}$ \\
\hline \hline 33,50 & 27,30 & 33,50 & 39,89 & 33,51 & 27,29 & 33,51 & 39,87 & 33,51 & 27,29 & 33,51 & 39,88 \\
- & $56^{\prime \prime}$ & - & $56^{\prime \prime}$ & - & $55,8^{\prime \prime}$ & - & $56,0^{\prime \prime}$ & - & $55,8^{\prime \prime}$ & - & $55,8^{\prime \prime}$
\end{tabular}


Bei jedem hergestellten Drucke war die Ablenkung sechsmal gemessen, dreimal nach rechts und dreimal nach links (durch Omschaltung des Motors). Die zweite Zeile (Tab. 2)

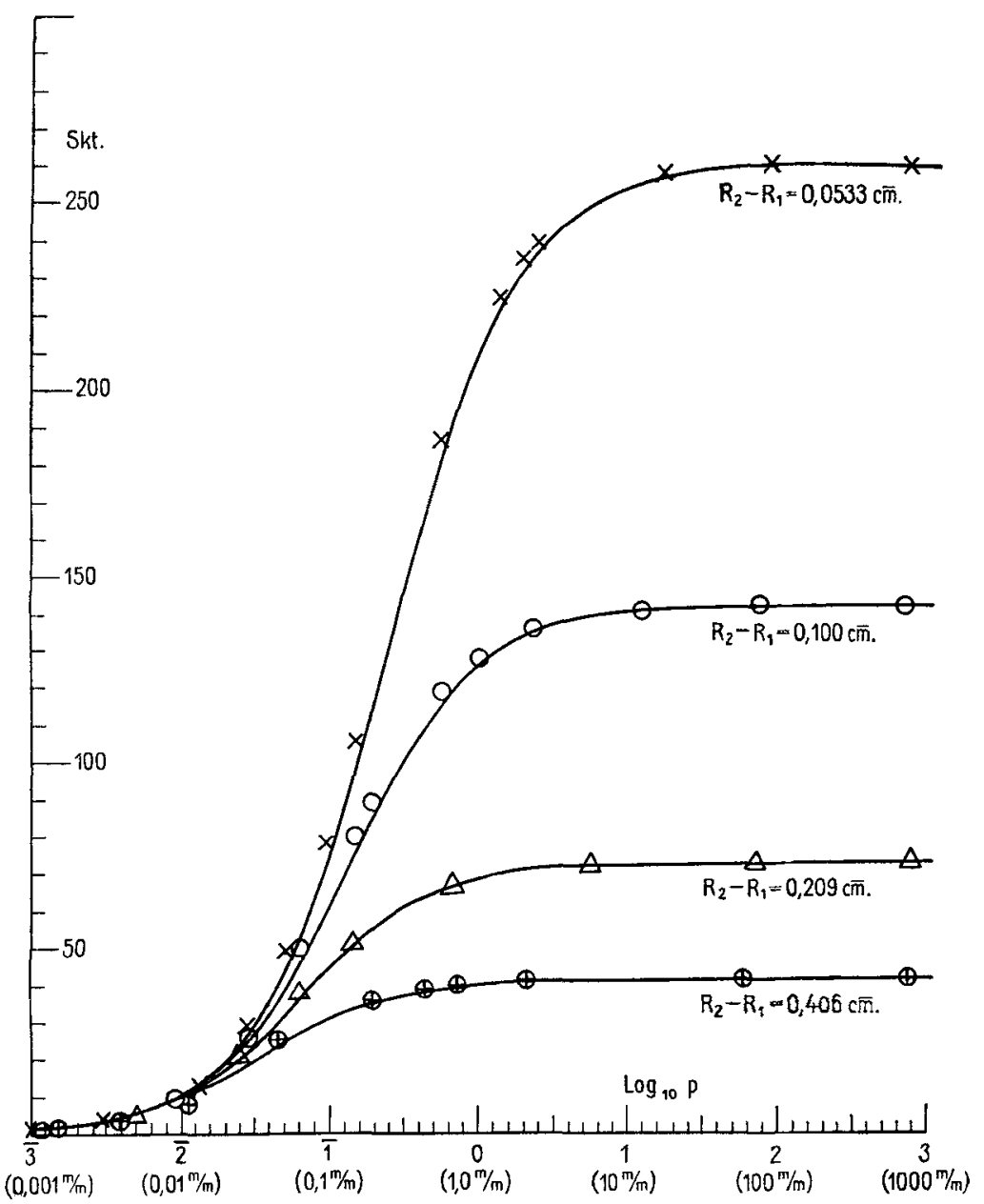

Fig. 5 .

gibt die Zeit für 48 Umdrehungen. Die Ablenkungen sind dann auf $60 \mathrm{Umdr}$./Min. reduziert und zum Mittelwert vereinigt. Die „berechneten" Kurven waren folgendermaben konstruiert: es war der Mittelwert des Temperatursprungs- 
koeffizienten aus Lasareffs ${ }^{1}$ ) Messungen entnommen, der Koeffizient $a_{0}$ nach der Formel (10) berechnet und mit Hilfe (18) die vier Kurven für die vier obengenannten Zylinder ermittelt. Die Ordinaten (Fig. 5) sind in Skalenteilen ausgedrückt und als Abszisse der Logarithmus des Druckes $p$ aufgetragen. Fig. 5 zeigt, inwieweit die Beobachtungen $(x \circ \Delta \oplus)$ mit den berechneten Werten (ausgezogene Kurven) übereinstimmen.

Im Bereich $(0,001-0,015 \mathrm{~mm})$ sind die Beobachtungen auf Quecksilberdampf korrigiert. Diese Korrektion ergibt sich folgendermaßen: trägt man im Diagramm die aus der Beobachtung direkt entnommenen und auf gleiche Tourenzahl reduzierten Ablenkungen von 0,001 bis $0,015 \mathrm{~mm}$ ein, so liegen die entsprechenden Punkte auf einer Geraden (Ablenkung als Funktion des Druckes), was vollkommen der Theorie entspricht (vgl. oben), nur schneiden diese Geraden (Fig. 6, Versuchsreihen

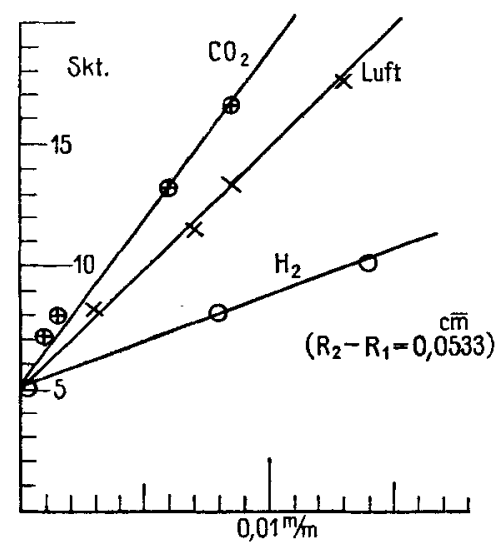

Fig. 6.

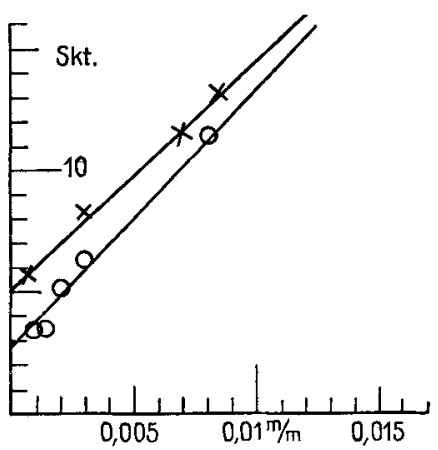

Fig. 7.

Nr. 45, 46, 47 Luft, Kohlensäure, Wasserstoff, Zylinder Nr. 4) die Ordinatenachse nicht im Nullpunkt, als ob wir mit wachsenden Verdünnungen einer Grenzablenkung uns nähern. Diese konstante Ablenkung ist am leichtesten durch den Quecksilberdampf zu erklären. $\mathrm{Da}$ im Intervall 0 bis $20^{\circ} \mathrm{C}$. die Tension des Quecksilbers sich rasch ändert, so war ein Versuch ausgeführt mit durch Schnee abgekühltem Quecksilber; die Korrektion fällt dann um ungefähr 40 Proz. kleiner aus, wie aus der Fig. 7

1) P. Lasareff, l. c. 
leicht ersichtlich ist, was eine wesentliche Stütze der obengenannten Hypothese bildet. AuBerdem zeigt die Rechnung, da $B$ der BewegungsgröBentransport durch den Quecksilberdampf beim Drucke, der der Tension des Dampfes bei $17^{\circ} \mathrm{C}$. entspricht, in Skalenteilen ausgedrückt gleich 4 (Skt.) sein muB. Die Rechnung ist auf Grund (11) unter Berücksichtigung des Molekulargewichts ausgeführt, dabei ist die für den Quecksilberdampf unbekannte GröBe 2-flf der für Luft gleich angenommenen, deshalb ist die Abweichung der berechneten GröBe 4 Skt. von der beobachteten 5 Skt. vollkommen zu erklären.

Bei größeren Drucken, falls man die gegenseitigen Stöße der Moleküle berücksichtigen muB, darf man diese Korrektion

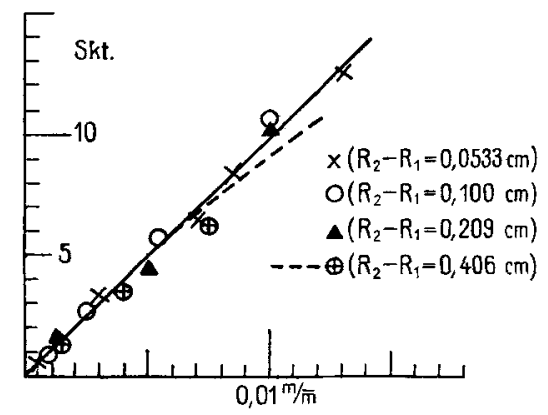

Fig. 8.

nicht anwenden, da aber der EinfluB des Druckes stark abnimmt, ist diese Korrektion überhaupt unnötig. Das ist vielleicht der Grund, weshalb der EinfluB des Quecksilberdampfes in den Temperatursprungmessungen, die bei höheren Drucken $(0,60-0,14 \mathrm{~mm})$ und für gröBere gegenseitige Entfernung der Platten $\left.(9 \mathrm{~mm})^{1}\right)$ ausgeführt waren, nicht zur Geltung kommt.

Die Ablenkungen bei kleinen Drucken sind unabbängig von der Größe $R_{2}-R_{1}$ (vgl. Fig. 5), was noch klarer aus der Fig. 8 hervorgeht. In diesem Druckgebiet ist der Bewegungsgrößentransport dem Gasdrucke proportional, was die Konstruktion eines, dem von $\mathrm{Knudsen}{ }^{\text {) }}$ ausgeführten „Absolutmanometer“ analogen, Instrumentes ermöglicht. ${ }^{3}$ )

1) P. Lasareff, l. c. p. 241.

2) M. Knudsen, Ann. d. Phys. 32. p. 809. 1910.

3) Versuche in dieser Richtung werden vom Verfasser weitergeführt. 


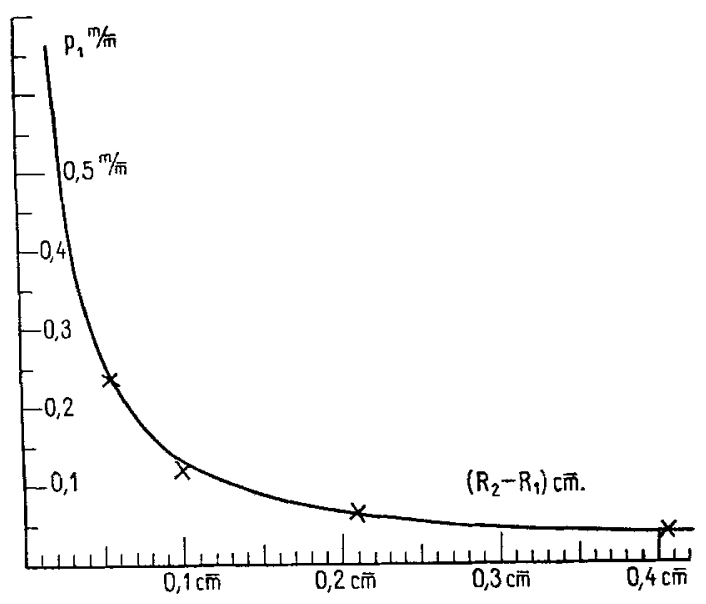

Fig. 9.

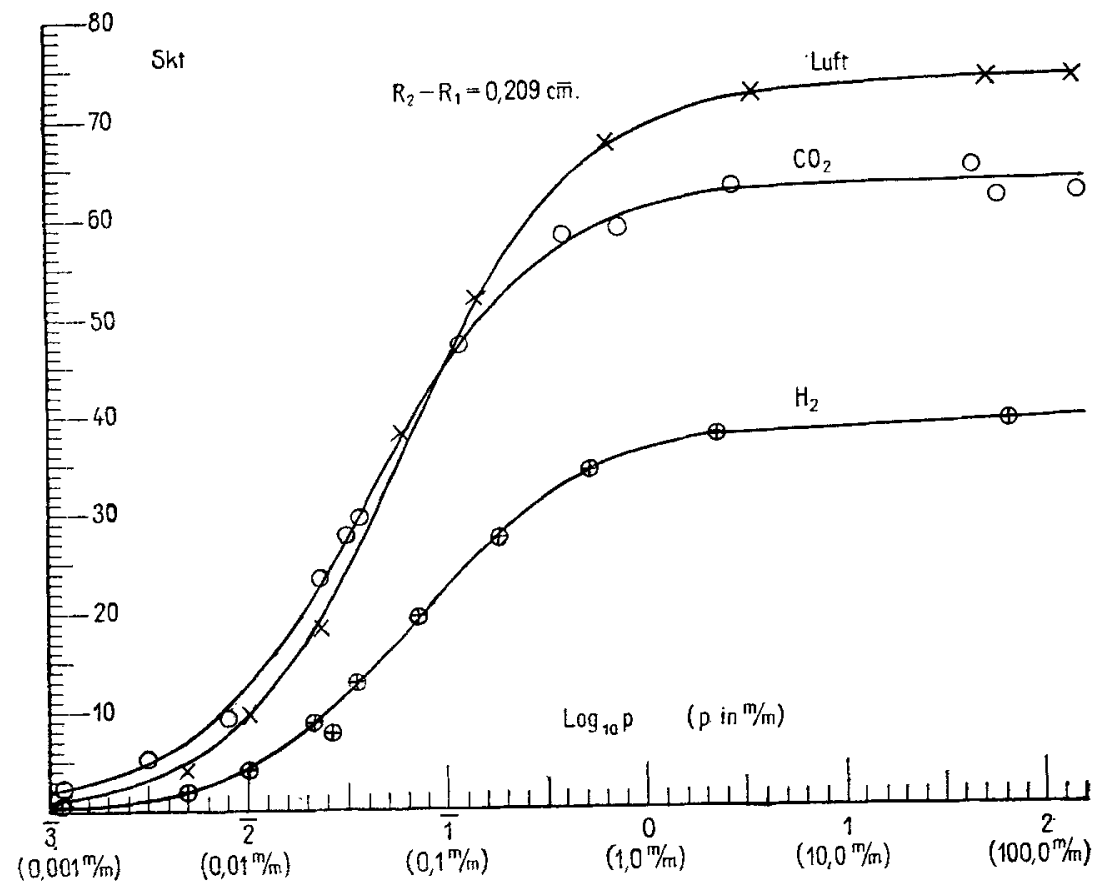

Fig. 10. 
Die Kurve Fig. 9 zeigt die theoretische, durch das Experiment bestätigte, von Prof. $P$. Lebedew vermutete $B e-$ ziehung zwischen dem Drucke $p$, der dem Inflexionspunkte der Kurve entspricht und der GröBe $R_{2}-R_{1}$ (vgl. 6"' und weiter).

Die Kurven (Fig. 10) zeigen den Gang der Erscheinung in verschiedenen Gasen (Luft, Kohlensäure, Wasserstoff) für Zylinder Nr. $2\left(R_{2}-R_{1}=0,209 \mathrm{~cm}\right)$, wobei die ausgezogenen Kurven für Luft und Kohlensäure aus den Temperatursprungsmessungen von Lasareff berechnet sind. Die Übereinstimmung, wie man leicht einsieht, ist eine recht gute. Die folgenden Tabellen stellen die Resultate zahlenmäßig dar.

Tabelle 3 .

Versuchsreihe Nr. 36. Luft. Zylinder Nr. 2.

\begin{tabular}{|c|c|c|c|}
\hline$p \mathrm{~mm}$ & $\log _{10} p$ & $a$ ber. & a beob. \\
\hline 750,0 & 2,875 & 73,2 & 73,3 \\
\hline 73,0 & 1,863 & 73,2 & 73,1 \\
\hline 3,75 & 0,574 & 72,0 & 72,2 \\
\hline 0,675 & $\overline{1}, 829$ & 67,0 & 67,3 \\
\hline 0,145 & $\overline{\mathbf{1}}, 161$ & 51,0 & 51,7 \\
\hline 0,061 & $\overline{2}, 785$ & 35,0 & $38,3 ?$ \\
\hline 0,023 & $\overline{2}, 365$ & 19,7 & 19,0 \\
\hline 0,0105 & $\overline{2}, 022$ & 9,5 & $10,0^{*}$ \\
\hline 0,005 & $\overline{\mathbf{3}}, 699$ & 5,1 & $4,2^{*}$ \\
\hline 0,0012 & $\overline{3}, 079$ & 1,0 & $1,3 *$ \\
\hline
\end{tabular}

Tabelle 4.

Versuchsreihe Nr. 37. $\mathrm{CO}_{\mathbf{9}}$. Zylinder Nr. 2.

\begin{tabular}{c|c|c|c}
\hline \hline$p \mathrm{~mm}$ & $\log _{10} p$ & $\alpha$ ber. & $\alpha$ beob. \\
\hline 750,0 & 2,875 & 62,8 & 61,4 \\
71,0 & 1,851 & 62,8 & 60,9 \\
48,0 & 1,681 & 62,8 & 64,5 \\
3,0 & 0,477 & 62,0 & 62,7 \\
0,765 & $\overline{1}, 884$ & 59,7 & 58,4 \\
0,405 & $\overline{1}, 608$ & 57,0 & 57,9 \\
0,0365 & $\overline{\mathbf{2}}, 562$ & 29,2 & 29,8 \\
0,0125 & $\overline{\mathbf{2}}, 097$ & 14,0 & $12,7^{*}$ \\
0,003 & $\overline{3}, 477$ & 4,5 & $5,0^{*}$ \\
0,0012 & $\overline{\mathbf{3}}, 079$ & 2,0 & $1,9^{*}$
\end{tabular}

Die mit * bezeichneten Werte sind auf Quecksilberdampf korrigiert. 
Nur mit Wasserstoff, für den aber die Temperaturursprungsmessungen bei verschiedenen Autoren stark auseinandergehen, habe ich keine befriedigende Übereinstimmung zwischen beobachteten und theoretischen Werten (aus dem Temperatursprung berechneten) gefunden, was aber den Verunreinigungen, die sicher in Gasen aus käuflichen Stahlzylindern vorhanden sind, zugeschrieben werden muB; deshalb ist die Kurve für Wasserstoff aus Experimenten direkt ermittelt.

\section{Zusammenfassung.}

1. Es wurde eine Methode der stationären Ablenkung ausgearbeitet, die eine genaue und bequeme Untersuchung der inneren Reibung im Druckintervall (760 bis $0,001 \mathrm{~mm}$ gestattet.

2. Es war auf Grund der Maxwell-Boltzmannschen gastheoretischen Untersuchungen der BewegungsgröBentransport $G$ als Funktion des Druckes berechnet. Diese Rechnung zeigt:

a) $\mathrm{DaB}$ bei gröBeren $\nabla$ erdünnungen eine Gleitung an der Oberfläche fester Körper auftritt, deren GröBe $a_{0} / p=c_{0} \lambda$ der mittleren freien Weglänge $\lambda$ direkt und somit dem Drucke $p$ umgekehrt proportional ist.

b) Der Gleitungskoeffizient $a_{0}$ steht mit dem von Smoluchowski eingeführten Temperatursprungskoeffizient $\gamma p$ in der einfachen Beziehung $a_{0}=\frac{8}{15} \gamma p$.

3. Es war auf Grund der Temperatursprungsmessungen die Gleitungskonstante $a_{0}$ berechnet und die theoretische Kurve $G$ (Bewegungsgrößentransport) als Funktion von $\log p$ konstruiert.

4. Die Versuche mit Luft und Kohlensäure stimmen mit den oben genannten berechneten Werten gut überein.

5. Ist die Verdünnung so weit getrieben, daB die mittlere freie Weglänge die Gasschichtdicke $d=R_{2}-R_{1}$ übertrifft, so ist der BewegungsgröBentransport von $d$ unabhängig, was nur durch Molekulartheorie erklärt werden kann.

6. Die Kurve $G=f(\log p)$ hat einen Inflexionspunkt, der bei Drucken $p_{1}$ auftritt, die umgekehrt proportional der Gasschichtdicke $d$ sind. Dieses Resultat war von Prof. P. Le bedew 
vermutet; die Rechnungen, sowie die Versuche haben diese Vermutung vollkommen bestätigt.

Die Anregung zu dieser Arbeit verdanke ich meinem verstorbenen Lehrer Prof. P. Lebedew und kann nicht ohne tiefste Empfindung des Dankes an das lebhafte Interesse, das mein hochverehrter Lehrer in den letzten Tagen seines Lebens dieser Untersuchung widmete, denken.

Es sei mir auch gestattet, an dieser Stelle dem Institut Solvey, der Ledenzoffschen Gesellschaft für Förderung der Experimentalwissenschaften und vielen Privatpersonen für die zugewandten nötigen Mittel, die eine weitere Tätigkeit des Lebede w schen Physikalischen Laboratoriums an der Städtischen Universität in Moskau ermöglichten, meinen wärmsten Dank auszusprechen.

Moskau, Lebedewsches Physikalisches Laboratorium an der Städtischen Universität, 21. Februar 1913.

(Eingegangen 3. März 1903.) 